\title{
Interaction of Rice Quantitative Trait Locus gw9.1 with Three Grain Shape Genes
}

\author{
Yun-Joo Kang, Yun-A Jeon, Ju-Won Kang, Hyun-Sook Lee, Sang-Nag Ahn* \\ Department of Agronomy, Chungnam National University, Daejeon 34134, Korea
}

\begin{abstract}
Grain size is one of the most important factors determining grain yield in rice breeding. In previous studies, we constructed high-density maps for two quantitative trait loci (QTL) for grain weight, $\operatorname{tg} w 2$ and gw9.1, using progeny derived from crosses between the japonica cultivar Hwaseong and Oryza grandiglumis, and Hwaseong and O. rufipogon (IRGC 105491), respectively. The wild alleles contributed an increase in grain weight at these two loci. We developed an $F_{2}$ population (146 plants) by crossing two near isogenic lines (NILs) harboring $\operatorname{tg} w 2$ and $g w 9.1$ to know how they interact in the near isogenic background. Simple sequence repeat markers tightly linked to two QTL were used to check the genotype of the $\mathrm{F}_{2}$ population. Based on the genotype at two loci, $146 \mathrm{~F}_{2}$ plants were classified into 9 groups with a combination of three genotypes at each two loci. Two gene interaction was not significant $(P=0.99)$ in the $\mathrm{F}_{2}$. Homozygous plants with wild alleles at two loci showed significantly higher 1,000 grain weight than plants with a single QTL in the $\mathrm{F}_{2}$ and $\mathrm{F}_{3}$. These results indicate that two QTLs act additively in distinct or complementary pathways in controlling GW. Gene expression analysis was also performed to know the relationship of the gw9.1 QTL with three major grain size genes with Hwaseong and two NILs plants at the transcription level. The results from this study provide insight into grain size regulation in rice and are likely to be useful for marker aided selection for grain size.
\end{abstract}

Keywords Rice, Grain weight, Quantitative trait loci, Interaction, Near isogenic line

\section{INTRODUCTION}

Rice (Oryza sativa L.) is one of the most important cereal food crop. Rice yield is closely related with grain weight. Grain weight shows a positive correlation with grain size which is measured by grain length (GL), grain width (GW), and grain thickness (GT). The identification of major quantitative trait loci (QTL) for grain shape is an important objective of rice genetic research and breeding program (Kang et al. 2011; Huang et al. 2013; Nagata et al. 2015). Extensive studies were carried out to identify QTL for grain size and nearly 200 QTL for GL and GW have been reported in rice (Nagata et al. 2015). More than ten genes to control grain size have been cloned and molecularly characterized, GS3 (Fan et al. 2006), GW2 (Song et al. 2007), qGL3 (Hu et al. 2012), GS5 (Li et al. 2011), qSW5 (Shomura et al. 2008), GW8 (Wang et al. 2012), HGW (Li et al. 2012), TGW6 (Ishimaru et al. 2013), GW6a (Song et al. 2015), and GS6 (Sun et al. 2013). GW2 encodes an E3 ubiquitin ligase and affects $\mathrm{GW}$ by restricting cell division. A significant increase of $49.8 \%$ in grain weight was observed in the near isogenic line (NIL)-GW2 compared with the control, FAZ1-NIL (Song et al. 2007). GS5 encodes a putative serine carboxypeptidase and functions as a positive factor for GW (Li et al. 2011). SPL16 (GW8) promotes cell division leading to increase in $\mathrm{GW}$ and showed a $13.9 \%$ advantage in 1,000 grain weight (TGW; Wang et al. 2012). These studies improved our understanding of genetic control over grain size traits; however the employment of these major QTL in practical breeding program for enhancing rice yield potential is hampered. This is because the information of additive and epistatic interactions across multiple grain size genes essential for combining favorable alleles resulting in enhanced traits is not sufficient for fine-tuning grain shape in practical breeding programs (Huang et al. 2013). In this regard, it is

Received January 4, 2016; Revised January 29, 2016; Accepted January 31, 2016; Published February 28, 2016

*Corresponding author Sang-Nag Ahn, ahnsn@cnu.ac.kr, Tel: +82-42-821-5728, Fax: +82-42-822-2631 
necessary to decipher the interactions among grain size genes and combine appropriate genes/QTLs.

QTL-NILs are promising materials to detect interactions among genes and to characterize gene actions in details because the background genetic effects on the analysis can be minimized in QTL-NILs. However, a few studies reported the employment of QTL-NIL for analyzing the interaction among grain size QTL. Jin et al. (2011) detected an epistatic interaction between two QTL, gw8.1 and gw9.1. Wang et al. (2012) also examined the interaction between GS3 and $q G W 8$ in a near-isogenic (HJX74) background. Lu et al. (2013) compared the grain size among haplotypes of GW2, GS3, qSW5, and GS5 in the Zhanshan97 background. However, detailed analysis of interaction among grain shape QTLs will provide more insights into the molecular mechanisms underlying seed size development in rice and is likely to be useful for gene pyramiding aimed at improving rice grain yield.

A
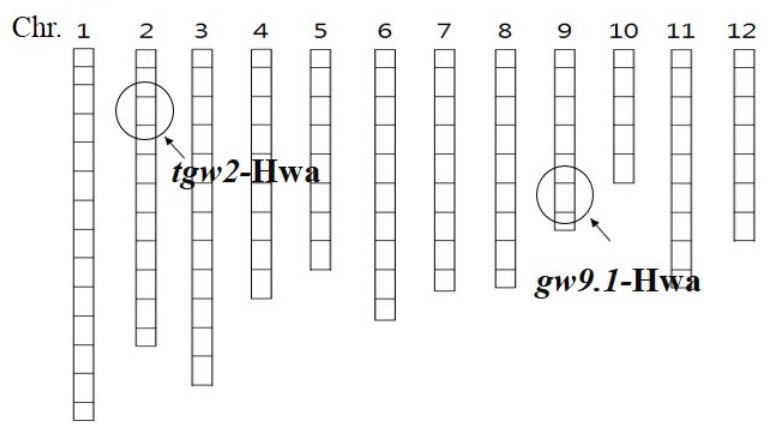

C

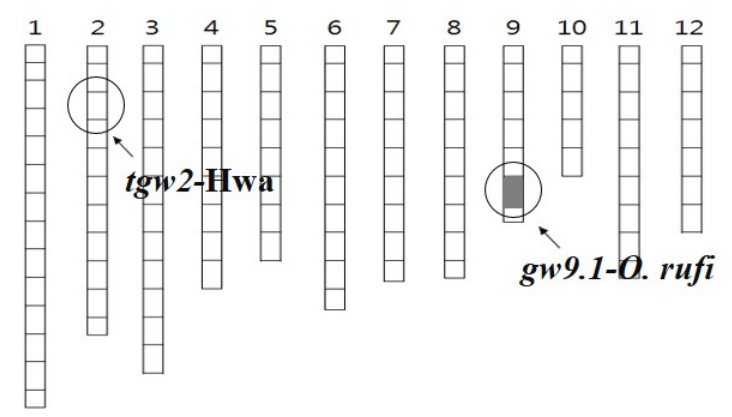

In our previous studies, two QTL-NILs, tgw2-NIL (Yoon et al. 2006) and gw9.1-NIL (Xie et al. 2008) related with grain size were developed by introgressing chromosomal segments of two wild species, O. grandiglumis (IRGC 101154) and O. rufipogon (IRGC 105491) into the Korean elite japonica cultivar Hwaseong, respectively. This study was carried out to elucidate the interaction of two QTL, tgw2 and gw9.1. Also, to know the relationship of the $g w 9.1$ QTL with three major grain size genes $G W 2$, $G S 3$, and $H G W$ at the transcription level, gene expression analysis was performed with three lines, Hwaseong and two NILs.

\section{MATERIALS AND METHODS}

\section{Plant materials}

Molecular marker-assisted backcrossing was used to

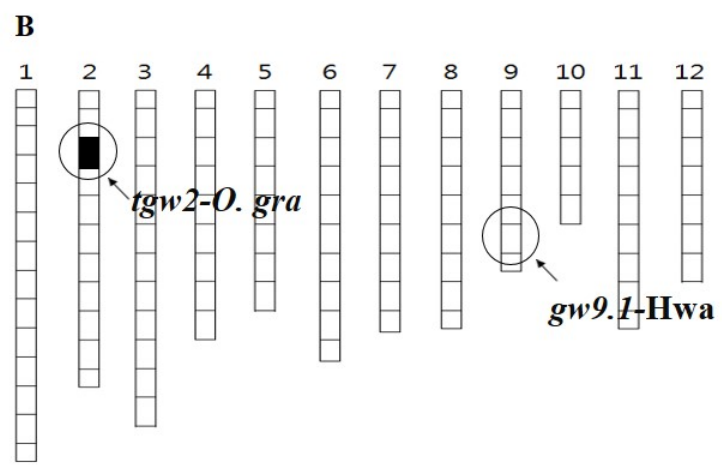

\section{D}

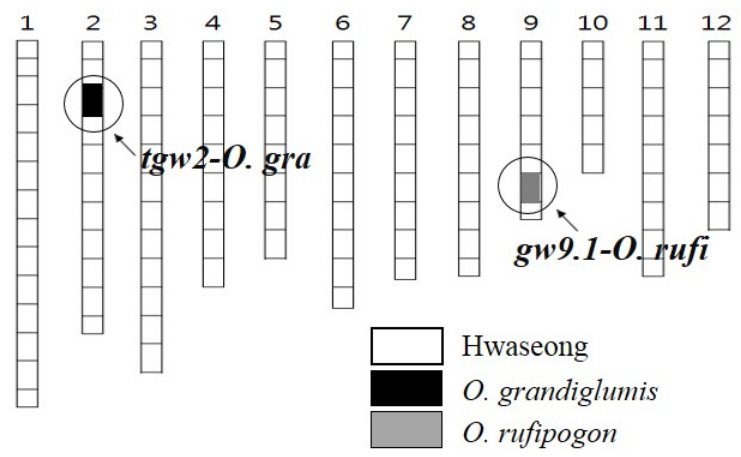

Fig. 1. Graphical genotypes of 4 representative groups. The two circles on the chromosomes represent regions for 2 quantitative trait loci. Open bars and solid bars in black show segments of the chromosomes derived from Hwaseong and Oryza grandiglumis $(O$. gra) or O. rufipogon $(O$. rufi). Each number on top of the bars are chromosome number. (A) Hwaseong (Hwa). (B) tgw2-NIL. (C) gw9.1-NIL. (D) tgw2+gw9.1-NIL. Chr.: chromosome, NIL: near isogenic line. 
pyramid two QTL, $\operatorname{tg} w 2$ and $g w 9.1 .146 \mathrm{~F}_{2}$ plants were produced from a cross between two QTL-NILs and genotyped using simple sequence repeat (SSR) markers tightly linked to the two QTL. Four $F_{2}$ plants with 1 of 4 types of QTL-NILs: Hwaseong (Hwaseong homozygous at both loci), tgw2-NIL (O. grandiglumis homozygous at tgw2 and Hwaseong homozygous at gw9.1), gw9.1-NIL (Hwaseong homozygous at tgw2 and $O$. rufipogon homozygous at $g w 9.1$ ), and $\operatorname{tg} w 2+g w 9.1$-NIL (O. grandiglumis and $O$. rufipogon homozygous at $t g w 2$ and $g w 9.1)$ were selected and advanced to $F_{3}$ lines (Fig. 1).

\section{Field trials and trait evaluation}

During the summer of 2013, $146 \mathrm{~F}_{2}$ plants were grown in the experimental farm, Chungnam National University, Daejeon, Korea at a density of $15 \times 30 \mathrm{~cm}$ (line $\times$ row). Seeds were sown on April 25 and transplanted to the field on May 29. To minimize border effect, seedlings of $F_{2}$ plants were planted in the middle, excluding the first five and last five lines in each row. $146 \mathrm{~F}_{2}$ plants were measured for $\mathrm{GL}$, width, thickness and TGW in brown rice. Fully-filled seeds were re-dried in an oven at $30^{\circ} \mathrm{C}$ for 24 hours. TGW was evaluated by measuring the weight of 250 randomly-selected brown rice; this method was performed in triplicates and the values were averaged to yield a single mean. Four $\mathrm{F}_{3}$ lines selected from the $F_{2}$ population were grown in the field during the summer of 2014. The planting density was the same as 2013. Four $F_{3}$ lines were evaluated for 11 traits (culm length [CL], panicle length [PL], panicle number [PN], spikelets per panicle [SPP], fertility [FER]). Each line was represented by a row of 30-day-old seedlings planted with $15 \mathrm{~cm}$ between plant and $30 \mathrm{~cm}$ between rows in a completely randomized block design with two replications. CL was measured in centimeters from the surface of soil to panicle neck. PL was measured in centimeters from panicle neck to the tip of panicle. PN was calculated as the number of panicles per plant. For SPP, all seeds on panicle were counted including unfilled seeds. For FER, filled seeds were counted and divided with SPP (\%). The middle 10 plants from each line were chosen for the evaluation, and the average of the measurements was used for the phenotype of each line for the TGW. Grains with hulls were allowed to dry naturally after harvesting, seeds were re-dried in an oven at $30^{\circ} \mathrm{C}$ for 24 hours. The TGW was evaluated by measuring the weight of 250 randomly selected brown rice per plant (10 plants per line). GL, GW, and GT were measured. GL, GW, and GT were measured for 15 grains (brown rice) per plant (10 plants per line) using a 150-mm Vernier caliper (Mitutoyo Corp., Kawasaki, Japan). TGW was corrected for the $15 \%$ grain moisture content. Amylose content (\%) and grain chalkiness were checked using a category of 0 (no chalky in the center and belly) to 9 (the grain opaque area $>75 \%$ ) (Juliano 1985). Means for two replications were evaluated for each trait and used in data analysis.

\section{DNA extraction and SSR analysis}

DNA was extracted from the fresh leaf tissue of each of $146 \mathrm{~F}_{2}$ plants. DNA extraction was performed as described in Causse et al. (1994). SSR analysis of the $\mathrm{F}_{2}$ population was performed according to the method described by Panaud et al. (1996). SSR markers tightly linked to two QTLs, RM12813 for tgw2 and ex9 for gw9.1 (Xie et al. 2008) were used for genotyping $146 \mathrm{~F}_{2}$ plants. Protocols for marker amplification using the polymerase chain reaction (PCR) and for size separation using polyacrylamide gel electrophoresis and marker detection using the silver staining procedure were as described in Xie et al. (2008). The silver staining kits were purchased from Bioneer Co., Daejeon, Korea (www.bioneer.co.kr).

\section{RNA extraction and gene expression analysis}

Total RNA was extracted from panicles, less than $1 \mathrm{~cm}$ long from plants around 23 days before heading; Hwaseong, tgw2-NILs, and gw9.1-NILs using Nucleospin RNA plant kit (MACHEREY-NAGEL, Düren, Germany). Sampling time and procedure followed those described in previous studies (Song et al. 2007; Mao et al. 2010; Li et al. 2012). The first-strand cDNA was synthesized using extracted RNA using a PrimeScript RT-PCR Kit (TaKaRa Biotechnology Co. Ltd, Tokyo, Japan). Gene expression was performed by quantitative Real-Time PCR (CFX Connect Real-Time System; Bio-Rad, Hercules, CA, USA) with $\mathrm{IQ}^{\mathrm{TM}} \mathrm{SYBR}^{\mathbb{R}}$ Green Supermix (BIO-RAD). Quantitative RT-PCR was done by using 3 steps protocol; $95^{\circ} \mathrm{C}$ for 1 minute for denaturation, 40 cycles of $95^{\circ} \mathrm{C}$ for 15 
seconds (denaturation), $55^{\circ} \mathrm{C}$ for 30 seconds (annealing) and $72^{\circ} \mathrm{C}$ for 30 seconds (extension), followed by final extension at $72^{\circ} \mathrm{C}$ for 7 minutes, and melting curve, temperature increased from $72^{\circ} \mathrm{C}$ to $95^{\circ} \mathrm{C}$ by $0.2^{\circ} \mathrm{C}$ for 5 seconds. For qRT-PCR primers, OsUBQ5 (Oryza sativa Ubiquitin 5) was used as control and three primers for qRT-GW2 (Yan et al. 2011; Lu et al. 2013), qRT-GS3 (Yan et al. 2011), and $H G W$ were designed (Table 1). Gene expression was performed in three replicates and the average values of three replicates were used in data analysis.

\section{Statistical analysis}

To analyze the interaction effect for grain weight, nine genotypes were identified from $146 \mathrm{~F}_{2}$ plants based on SSR genotypes tightly linked to each target QTL. In the $\mathrm{F}_{2}$ generation, QTL was declared if the phenotype was associated with a marker locus at $P<0.05$ using one-way ANOVA. Two-way ANOVA was used to analyze the epistatic interaction between two QTL. The proportion of observed phenotypic variation attributable to a particular QTL was estimated by the coefficient of determination
$\left(R^{2}\right)$. The mean values of four QTL-NILs in the $\mathrm{F}_{3}$ were compared using Duncan's multiple range test using SAS 9.3 program (SAS Institute, Cary, NC, USA).

\section{RESULTS}

\section{QTL Confirmation}

The existence of two QTL was confirmed by QTL analysis of the $\mathrm{F}_{2}$ population (Table 2, Fig. 2). The $O$. grandiglumis and $O$. rufipogon alleles increased grain weight in the Hwaseong background which is consistent with those in earlier reports (Yoon et al. 2006; Xie et al. 2008). The genotype-based distribution of the grain weight in the $\mathrm{F}_{2}$ at the two loci is shown in Fig. 2. Grain weight of two NILs for $\operatorname{tg} w 2$ and $g w 9.1$ was $29.6 \mathrm{~g}$ and $24.7 \mathrm{~g}$, respectively, and significantly higher than that of Hwaseong-NIL $(23.0 \mathrm{~g})(P<0.05)$. At the tgw 2 locus, the mean TGW of the homozygous $O$. grandiglumis class was significantly higher than those of the heterozygous and Hwaseong homozygous class. There was also a significant difference between heterozygote plants and Hwaseong

Table 1. Primers for quantitative RT-polymerase chain reaction.

\begin{tabular}{|c|c|c|c|}
\hline \multicolumn{2}{|c|}{ Primer ${ }^{2)}$} & Sequences $\left(5^{\prime} \rightarrow 3^{\prime}\right)^{\mathrm{y})}$ & Predicted size (bp) \\
\hline \multirow[t]{2}{*}{ OsUBQ5 } & $\mathrm{F}$ & GAAGTAAGGAAGGAGGAGGA & \multirow{2}{*}{100} \\
\hline & $\mathrm{R}$ & AAGGTGTTCAGTTCCAAGG & \\
\hline \multirow[t]{2}{*}{$\mathrm{qRT} \_G W 2$} & $\mathrm{~F}$ & CTGCAGCAGGGAAGTGGT & \multirow{2}{*}{96} \\
\hline & $\mathrm{R}$ & ATGTCTGAGCCCGCGTAG & \\
\hline \multirow[t]{2}{*}{ qRT_GS3 } & $\mathrm{F}$ & CATCGGAGAAGCGAAGTCA & \multirow{2}{*}{109} \\
\hline & $\mathrm{R}$ & CAGCAGCAGATCCAGGAGA & \\
\hline \multirow[t]{2}{*}{$H G W$} & $\mathrm{~F}$ & AGAACCCCAACTGGAGCAAG & \multirow{2}{*}{118} \\
\hline & $\mathrm{R}$ & ACTGGATCCCCTGGAGTAGC & \\
\hline
\end{tabular}

${ }^{\mathrm{z})} \mathrm{F}$ : forward, R: reverse.

${ }^{\mathrm{y})}$ All sequences are based on cDNA sequences.

Table 2. QTLs detected for grain weight based on one-way ANOVA in the $F_{2}$ population.

\begin{tabular}{|c|c|c|c|c|c|c|c|c|}
\hline \multirow{2}{*}{ Cross } & \multirow{2}{*}{$\mathrm{QTL}^{\mathrm{z})}$} & \multirow{2}{*}{ Chr. } & \multirow{2}{*}{ Marker } & \multirow{2}{*}{$P$-value } & \multirow{2}{*}{$\mathrm{R}^{2}$} & \multicolumn{3}{|c|}{ Mean } \\
\hline & & & & & & $\mathrm{HH}$ & GH/HR & GG/RR \\
\hline \multirow[t]{2}{*}{$\operatorname{tg} w 2 / g w 9.1$} & $\operatorname{tg} w 2$ & 2 & RM12813 & $<0.0001$ & 0.73 & $22.3 b^{y)}$ & $24.3 b$ & $26.8 \mathrm{a}$ \\
\hline & $g w 9.1$ & 9 & ex9 & 0.01 & 0.39 & $22.3 b$ & $22.8 \mathrm{ab}$ & $23.9 \mathrm{a}$ \\
\hline
\end{tabular}

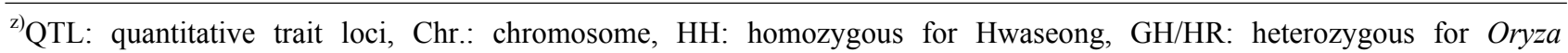
grandiglumis or O. rufipogon, GG/RR: homozygous for O. grandiglumis or O. rufipogon.

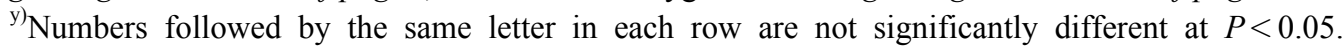



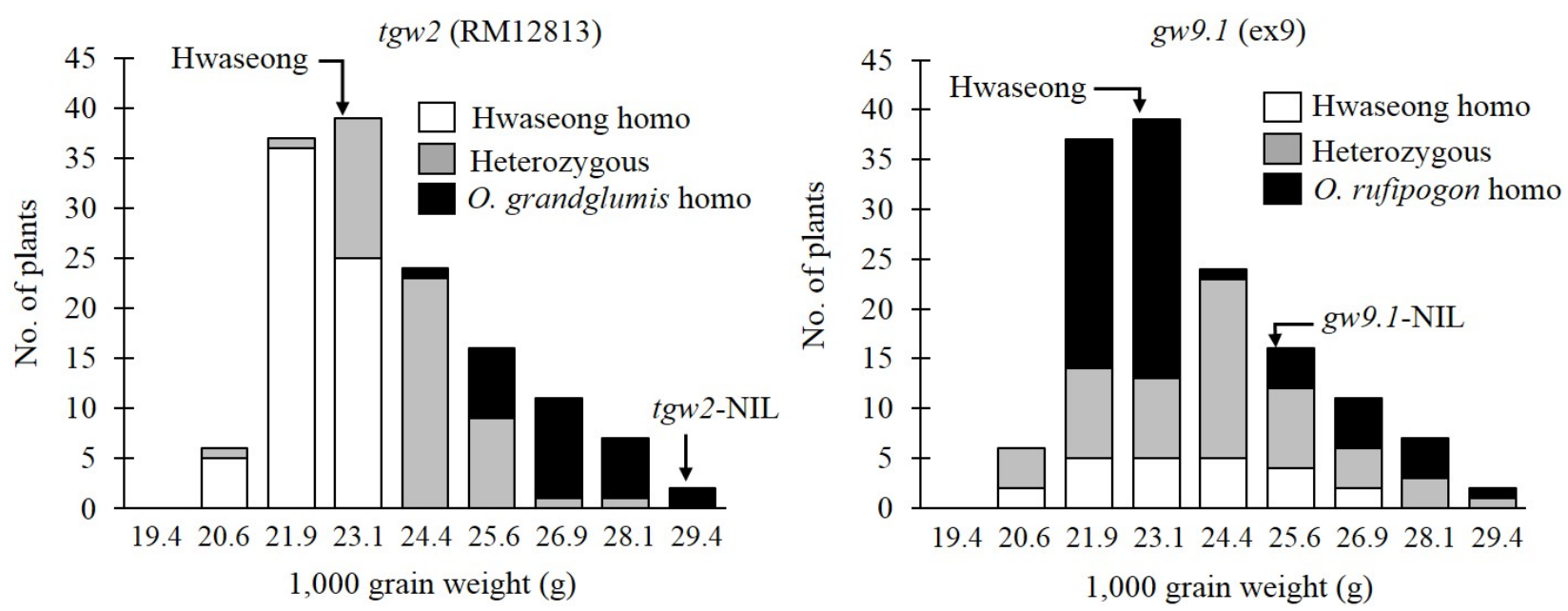

Fig. 2. Frequency distribution of 1,000 grain weight in 3 genotype classes in an $F_{2}$ population. NIL: near isogenic line, O.: Oryza.

homozygote plants (Table 2). This result indicated that the O. grandiglumis allele increased TGW in the Hwaseong background in an additive fashion.

TGW of the homozygous $O$. rufipogon class at $g w 9.1$ was significantly higher than the Hwaseong homozygous class. However, no significant difference between TGW of the homozygous $O$. rufipogon class and the heterozygous class at $g w 9.1$ was detected. The finding that $\operatorname{tg} w 2$ and gw9.1 explained $73.5 \%$ and $38.8 \%$ of the phenotypic variance for TGW in the $F_{2}$, respectively confirms the existence of the two QTLs (Table 2).

\section{Pyramiding two grain weight QTL}

Based on the genotypes at the two loci, RM12813 for $\operatorname{tg} w 2$ and ex 9 for $g w 9.1,146 \mathrm{~F}_{2}$ plants were classified into nine groups with a combination of three genotypes (homozygous for Hwaseong, O. rufipogon/O. grandiglumis or heterozygous) at each locus. To test the pyramiding effect of the two QTL, the average grain weight of nine genotype classes in the $F_{2}$ population was compared. Digenic interaction was not detected based on two-way ANOVA ( $P=0.99$ ) (Fig. 3 ). The TGW increasing effect of the $O$. grandiglumis allele was observed in three genotype classes at the tgw2 locus. Also, the $O$. rufipogon allele increased TGW in three genotype classes at the gw9.1 locus. The highest grain weight was observed in genotype class homozygous for $O$. grandiglumis and $O$. rufipogon at

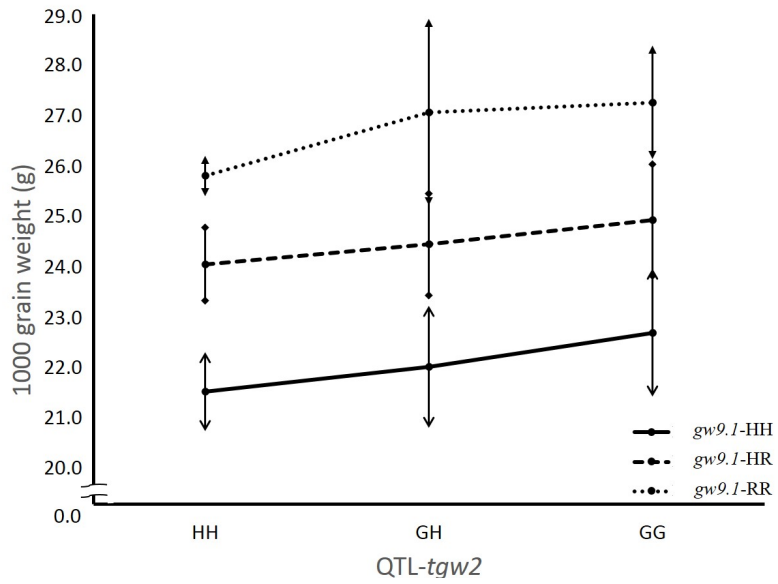

Fig. 3. Differences in 1,000 grain weight for different genotype classes between $\operatorname{tg} w 2$ and $g w 9.1$ in the $F_{2}$ population from the cross between two quantitative trait loci (QTL)-near isogenic lines (NILs) tgw2-NIL and gw9.1-NIL. HH: homozygous for Hwaseong, GH/HR: heterozygous for Oryza grandiglumis or O. rufipogon, GG/RR: homozygous for O. grandiglumis or O. rufipogon.

both loci $\operatorname{tg} w 2$ and $g w 9.1$ indicating no epistatic interaction between $\operatorname{tg} w 2$ and $g w 9.1$. To further confirm this finding that no interaction exists between $\operatorname{tg} w 2$ and $g w 9.1$, grain weight of four QTL-NILs was measured in the $\mathrm{F}_{3}$ (Table 3). Four $F_{3}$ lines were selected from the $F_{2}$ population based on the genotypes at two loci (Fig. 1). The effect of the $O$. 
Table 3. Comparison of 11 traits among 4 different groups at $F_{3}$ generation.

\begin{tabular}{|c|c|c|c|c|c|c|c|c|c|c|c|}
\hline \multirow{2}{*}{ Line } & \multicolumn{11}{|c|}{ Trait } \\
\hline & $\mathrm{CL}^{\mathrm{z})}$ & PL & $\mathrm{PN}$ & SPP & FER & GL & GW & GT & TGW & $\mathrm{CH}$ & $\mathrm{AC}$ \\
\hline Hwaseong & $92 \mathrm{a}$ & $19.3 \mathrm{a}$ & $13 a$ & $102 \mathrm{a}$ & $92.0 \mathrm{a}$ & $5.29 \mathrm{c}$ & $2.87 \mathrm{c}$ & $2.17 \mathrm{~b}$ & $23.0 \mathrm{~d}$ & 0 & $20.1 b$ \\
\hline $\operatorname{tg} w 2-\mathrm{NIL}$ & $90 \mathrm{a}$ & $17.8 b$ & $11 b$ & $100 \mathrm{a}$ & $84.3 b$ & $5.37 \mathrm{~b}$ & $3.18 \mathrm{~b}$ & $2.35 \mathrm{a}$ & $29.6 b$ & 4 & $24.1 \mathrm{a}$ \\
\hline$g w 9.1-\mathrm{NIL}$ & $90 \mathrm{a}$ & $20.2 \mathrm{a}$ & $13 \mathrm{a}$ & $105 a$ & $92.9 \mathrm{a}$ & $5.36 \mathrm{~b}$ & $3.10 \mathrm{~b}$ & $2.20 \mathrm{~b}$ & $24.7 \mathrm{c}$ & 1 & $20.9 b$ \\
\hline $\operatorname{tg} w 2+\operatorname{g} w 9.1-\mathrm{NIL}$ & $91 \mathrm{a}$ & $19.3 \mathrm{a}$ & $11 b$ & $98 \mathrm{a}$ & $85.4 b$ & $5.42 \mathrm{a}$ & $3.31 \mathrm{a}$ & $2.40 \mathrm{a}$ & $31.8 \mathrm{a}$ & 4 & $24.2 \mathrm{a}$ \\
\hline
\end{tabular}

Numbers followed by the same letter in each column are not significantly different at $P<0.05$.

${ }^{2)} \mathrm{CL}$ : culm length, PL: panicle length, PN: panicle number, SPP: spikelets per panicle, FER: fertility, GL: grain length, GW: grain width, GT: grain thickness, TGW: 1,000 grain weight, $\mathrm{CH}$ : chalkiness, AC: amylose content, NIL: near isogenic line.
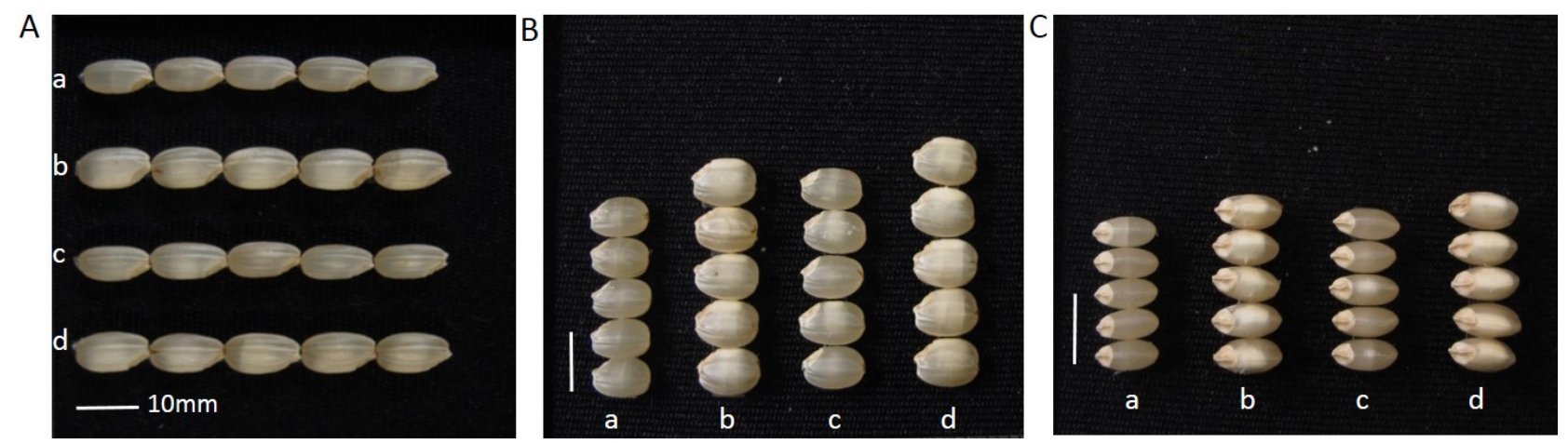

Fig. 4. Comparison of the grain shape traits among 4 groups. (A) Grain length. (B) Grain width. (C) Grain thickness. a: Hwaseong, b: tgw2-near isogenic line (NIL), c: $g w 9.1-\mathrm{NIL}, \mathrm{d}: \operatorname{tg} w 2+g w 9.1-\mathrm{NIL}$. White bar on picture indicates $10 \mathrm{~mm}$.

rufipogon allele at $g w 9.1$ on TGW was observed based on comparison of grain weight between Hwaseong and gw9.1-NIL. The effect of the O. grandiglumis allele at $\operatorname{tg} w 2$ on TGW was observed based on comparison of grain weight between Hwaseong and tgw2-NIL plants. Also, the additive effect of the $O$. grandiglumis allele at $\operatorname{tg} w 2$ and the O. rufipogon allele at $g w 9.1$ on TGW was observed based on comparison of grain weight between tgw2-NIL and $\operatorname{tg} w 2+g w 9.1$-NIL. TGW difference between $\operatorname{tg} w 2$-NIL and $\operatorname{tg} w 2+g w 9.1$-NIL caused by the genotype of $g w 9.1$ was observed. The finding indicates that there is no interaction between $\operatorname{tg} w 2$ and $g w 9.1$. To clarify which grain shape trait was associated with the increase in TGW, NILs were evaluated for GL, GW, and GT (Table 3, Fig. 4). GL and width of $\operatorname{tg} w 2+g w 9.1$-NIL plants were significantly higher than those of $\operatorname{tg} w 2$-NIL and $g w 9.1$-NIL plants. A phenotypic difference between $\operatorname{tg} w 2$-NIL and $\operatorname{tg} w 2+g w 9.1$-NIL caused by the $O$. rufipogon allele at $g w 9.1$ was observed. Similar results were observed for GT: grains of the tgw2-NIL and $\operatorname{tg} w 2+g w 9.1$-NIL plants were significantly thicker than gw9.1-NIL grains. These results indicate that the variation in grain weight in gw9.1-NIL is associated with GL and width whereas three grain shape traits are responsible for the variation in GW in tgw2-NIL.

\section{Impact of QTL pyramiding on agronomic traits}

To know the effect of the chromosomal segment harboring tgw2 and gw9.1 from $O$. grandiglumis and $O$. rufipogon on agronomic traits, NILs were evaluated for 11 agronomic traits (Table 3). Results showed that no significant difference was observed for CL and SPP. PL was significantly higher in $g w 9.1$-NIL than the other NILs and Hwaseong. However, tgw2-NIL and $\operatorname{tg} w 2+g w 9.1-\mathrm{NIL}$ plants had significantly lower number of panicles per plant 
than Hwaseong and this result seems to imply that QTL associated with panicle number is located in the $O$. grandiglumis introgression on chromosome 2 and is consistent with the previous study (Yoon et al. 2006). Also, $\operatorname{tg} w 2$-NIL and $\operatorname{tg} w 2+g w 9.1$-NIL plants displayed higher ratio of grain chalkiness and amylose content than Hwaseong.

\section{Gene expression}

To investigate whether gw9.1 interacts with other three grain size genes, $G W 2, G S 3$, and $H G W$, we performed the

(A)
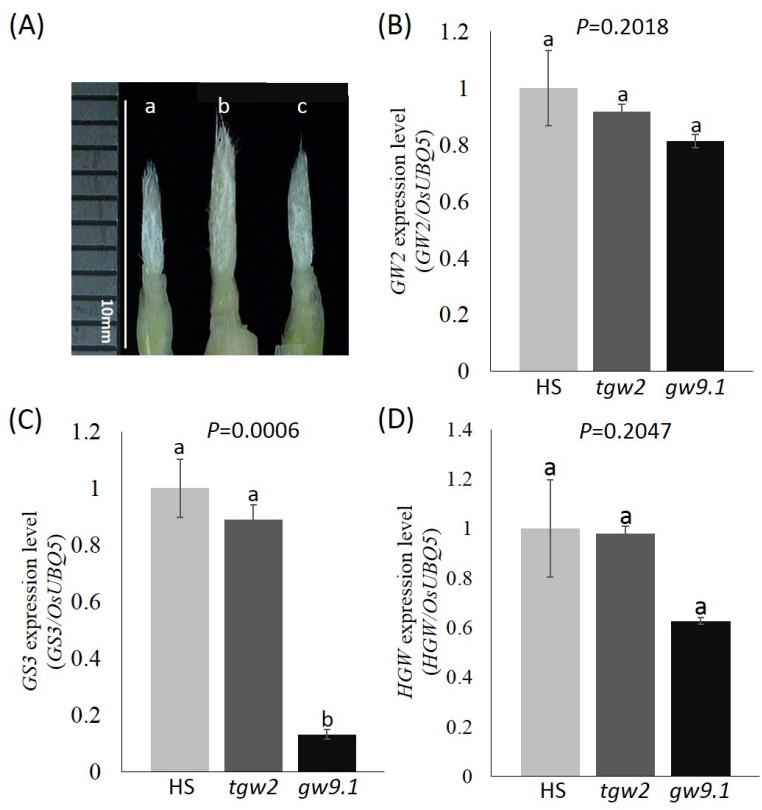

Fig. 5. Gene expression level of 3 different grain shape genes in Hwaseong (HS), tgw2-near isogenic line (NIL), and gw9.1-NIL. (A) Panicle samples for RNA extraction. White bar indicates $10 \mathrm{~mm}$. Samples sizes are less than $1 \mathrm{~cm}$. (B) $G W 2$, (C) $G S 3$, and (D) $H G W$. OsUBQ5 was used as normalization control for the RNA level. Error bars indicate standard deviation of three replicates. Each expression was repeated three times. One way ANOVA and Duncan's multiple range test were done with each stage in $G W 2$, $G S 3$, and $H G W$ at the significant level of $P=0.05$. Numbers followed by the same letter above the error bar are not significantly different at the $P=0.05$ based on Duncan's multiple range test. a: HS, b: $\operatorname{tg} w 2-\mathrm{NIL}, \mathrm{c}: \operatorname{gw9}$.1-NIL. quantitative RT-PCR with three groups: Hwaseong, tgw2-NIL and gw9.1-NIL (Fig. 5). For GW2 expression (Fig. 5B), three groups did not show any significant difference supporting the results from phenotypic data that $\operatorname{tg} w 2$ and $g w 9.1$ act in an additive manner. A significant difference was detected in GS3 expression level between Hwaseong and $g w 9.1$-NIL, and $\operatorname{tg} w 2$-NIL and $g w 9.1$-NIL (Fig. 5C). The difference between Hwaseong and $\operatorname{tg} w 2-\mathrm{NIL}$ in the expression level of $G S 3$ was observed $(P=0.189)$ although it did not reach the significance level $(P<0.05)$. The expression of $G S 3$ in $g w 9.1$-NIL was remarkably reduced compared to other two groups. This result might imply that the expression of GS3 may be negatively regulated by $g w 9.1$. However additional experiments are needed to confirm the effect of $g w 9.1$ on the expression of $G S 3$ because the molecular function of $g w 9.1$ is not known and gw9.1-NIL harbors several O. rufipogon genes in the introgressed segment (Xie et al. 2008). It is speculated that GS3 expression was affected by other $O$. rufipogon genes in the introgression in gw9.1-NIL. To analyze the interaction between $g w 9.1$ and $G S 3$, cloning of the gw9.1 gene is necessary. No significant difference among three groups was observed in $H G W$ expression (Fig. 5D).

\section{DISCUSSION}

Cloning and molecular characterization of several major QTL for grain shape traits have advanced our understanding of the grain size regulation in rice. However, the complicated additive and epistatic interactions among major grain size genes and some minor QTL are still unclear, and these impede the process of gene pyramiding program to attain higher grain yield in rice (Huang et al. 2013). In rice, primary mapping populations have been extensively used in QTL analyses (Yano and Sasaki 1997). It would be more difficult to determine the true genetic action of the QTL because the segregation of other QTLs often affects the genetic parameters of a given QTL in primary mapping population (Yano and Sasaki 1997). Only a limited number of interactions between QTLs were detected because primary populations cannot provide 
much information about the real nature of interactions among QTLs due to background noise. For example, two studies reported different results on the interaction between QTLs for grain size. Major QTL, GW2, and $q S W 5 / G W 5$ for grain size were detected using an $\mathrm{F}_{2}$ population from a cross between 'FAZ1' and 'Jizi1560' and no significant interaction was observed between three QTL, GW2, GS5, and $q S W 5 / G W 5$ (Ying et al. 2012). However, gene expression analysis with GW2-RNAi lines and CSSL of $q S W 5$ at the transcriptional level clearly showed that $q S W 5$ can be down-regulated by repression of $G W 2$ transcription implying that $G W 2$ might work upstream of $q S W 5$ (Yan et al. 2011). Also, in an association study using japonica rice, a significant difference in GL was detected between two $G W 2$ genotypes in the presence of nonfunctional GS3-A alleles, and the result indicated that two QTL, GW2 and GS3 interact each other (Lu et al. 2013).

QTL-NIL could provide a solution to this problem because the background genetic effects on the analysis can be minimized by QTL-NIL or populations derived from crosses between QTL-NILs with a uniform genetic background. Jin et al. (2011) detected an epistatic interaction between $g w 8.1$ and $g w 9.1$ using four QTL-NILs with allelic combination of $g w 8.1$ and $g w 9.1$ in a near-isogenic (Hwaseong) background. Wang et al. (2012) also developed four contrasting allelic combinations of GS3 and $q G W 8$ in a near-isogenic (HJX74) background and examined the interaction between $G S 3$ and $q G W 8$. NIL-GW8/gs3 plants harboring the qgs 3 allele from Basmati385 had grains heavier and longer than those from NIL-GW8/GS3 control plants and this result implies that two QTL act in an additive manner. These results clearly indicate that utilization of QTL-NIL is a powerful strategy for analyzing the gene interaction of QTLs.

In this study, we described the pyramided effect of $g w 9.1$ with $\operatorname{tg} w 2$ on agronomic traits. The results indicated that $\operatorname{tg} w 2$ showed no interaction with another grain weight gene, $g w 9.1$. Compared with TGW of Hwaseong, tgw2 from $O$. grandiglumis increased GW by up to $28 \%, g w 9.1$ from $O$. rufipogon by up to $7.4 \%$, and both by up to about $38.3 \%$. TGW of three QTL-NILs, tgw2-NIL, gw9.1-NIL and $\operatorname{tg} w 2+g w 9.1$-NIL were significantly higher than that of Hwaseong. TGW of $\operatorname{tg} w 2+g w 9.1$-NIL plants was signi- ficantly higher than those of $g w 9.1$-NIL and $\operatorname{tg} w 2$-NIL plants. These results indicate that two QTLs act additively in distinct or complementary pathways in controlling grain weight. Analysis of grain shape traits suggests that the increase in GL might be responsible for increased grain weight in $g w 9.1$-NIL whereas the increase in GL, width and thickness is associated with increased weight in $\operatorname{tg} w 2$-NIL and $\operatorname{tg} w 2+g w 9.1$-NIL. A drastic increase in grain size due to $\operatorname{tg} w 2$ did not increase grain yield proportionally in $\operatorname{tg} w 2$-NIL and $\operatorname{tg} w 2+g w 9.1$-NIL plants owing to decrease in grain filling and panicles per plant (data not shown) and this is a typical example of imbalance between the sink and source potentials (Takai et al. 2013). Also, grain quality traits (high rate of white center and white belly, higher amylose content) were observed in tgw2-NIL and $t g w 2+g w 9.1$-NIL plants compared to Hwaseong. It has to be determined whether this deleterious effect is due to a pleiotropy of the $t g w 2$ QTL or its linkage with other genes. This result suggests that breeders should practice caution when choosing QTL alleles for grain shape with pleiotropy or tight linkage with genes for deleterious effects.

We performed the quantitative RT-PCR with three groups, Hwaseong, tgw2-NIL, and gw9.1-NIL to investigate the interactions among grain size genes, GW2, GS3, and $H G W$. As for $G W 2$ expression, no significant difference among three groups was detected supporting the result of the phenotype data. For GS3 expression, the difference between Hwaseong and $\operatorname{tg} w 2$-NIL was observed $(P=0.189)$ although it did not reach the significance level $(P<0.05)$. This result is not consistent with the results from the study by Yan et al. (2011) reported that GS3 was positively regulated by $G W 2$ based on the gene expression level of $G W 2$ in both RNAi-GS3 and RNAi-GW2. Additional experiments are needed to analyze their interactions using QTL-NILs in more details at the phenotypic level. A significant difference between Hwaseong and $g w 9.1$-NIL, and $t g w 2-\mathrm{NIL}$ and $g w 9.1$-NIL were observed and these results suggest a possibility that gw9.1 might affect the expression of $G S 3$. For $H G W$, there was no significant difference among three groups. Li et al. (2012) analyzed the $G W 2$ expression level in $H G W$ mutants and found that the $G W 2$ expression level in $H G W$ mutants were significantly reduced compared to wild type 
(Zhounghua 11). The finding from this study that no significant difference between Hwaseong and $\operatorname{tg} w 2$-NIL in $H G W$ expression might support the finding that $H G W$ might be a upstream regulator of GW2 (Li et al. 2012).

To elucidate the interaction of two QTLs, gw9.1 and $\operatorname{tg} w 2$, an $\mathrm{F}_{2}$ population was developed by crossing two NILs harboring tgw2 and $g w 9.1$. SSR markers tightly linked to two QTLs were used to check the genotype of the $F_{2}$ population and to select four $F_{3}$ QTL-NILs with a combination of two QTLs. Two-way ANOVA revealed no interaction between 2 QTLs in the $\mathrm{F}_{2}$ population. TGW of homozygous plants with wild alleles at two loci was significantly higher than that of plants with a single QTL in the $F_{2}$ and $F_{3}$. These results indicate that two QTLs act additively in distinct or complementary pathways in controlling grain weight. Although the double-QTLs line gave further advantage in increasing TGW in the Hwaseong background, an increase in grain weight did not result in an increase in grain yield. However, it should be cautioned that grain filling and grain quality traits of the double-QTLs plants were decreased compared to Hwaseong indicating that $t g w 2$ may have some effect on plant morphology. It has to be determined whether this deleterious effect is due to a pleiotropy of the $\operatorname{tg} w 2 \mathrm{QTL}$ or linkage of $\operatorname{tg} w 2$ with other genes.

\section{ACKNOWLEDGEMENTS}

This work was carried out with the support of "Golden Seed Project" (Project No. PJ00993301), Ministry of Agriculture, Food and Rural Affairs (MAFRA), Ministry of Oceans and Fisheries (MOF), Rural Development Administration (RDA) and Korea Forest Service (KFS), Republic of Korea.

\section{REFERENCES}

Causse MA, Fulton TM, Cho YG, Ahn SN, Chunwongse J, $\mathrm{Wu} \mathrm{K}$, et al. 1994. Saturated molecular map of the rice genome based on an interspecific backcross population. Genetics 138: 1251-1274.
Fan C, Xing Y, Mao H, Lu T, Han B, Xu C, et al. 2006. GS3, a major QTL for grain length and weight and minor QTL for grain width and thickness in rice, encodes a putative transmembrane protein. Theor. Appl. Genet. 112: 1164-1171.

Hu Z, He H, Zhang S, Sun F, Xin X, Wang W, et al. 2012. A Kelch motif-containing serine/threonine protein phosphatase determines the large grain QTL trait in rice. J. Integr. Plant Biol. 54: 979-990.

Huang R, Jiang L, Zheng J, Wang T, Wang H, Huang Y, et al. 2013. Genetic bases of rice grain shape: so many genes, so little known. Trends Plant Sci. 18: 218-226.

Ishimaru K, Hirotsu N, Madoka Y, Murakami N, Hara N, Onodera $\mathrm{H}$, et al. 2013. Loss of function of the IAA-glucose hydrolase gene TGW6 enhances rice grain weight and increases yield. Nat. Genet. 45: 707-711.

Jin FX, Ji SD, Xie XB, Kang JW, Ju HG, Ahn SN. 2011. Detection of epistatic interaction of two QTLs, gw8.1 and gw9.1, underlying grain weight using nearly isogenic lines in rice. Jpn. J. Breed. 61: 69-75.

Juliano BO. 1985. Rice : chemistry and technology. 2nd ed. St. Paul, Minn.: American association of cereal chemists.

Kang JW, Yang P, Yun YT, Ahn SN. 2011. Mapping grain weight QTL Using near isogenic lines from an interspecific cross. Korean J. Breed. Sci. 43: 225-231.

Li J, Chu H, Zhang Y, Mou T, Wu C, Zhang Q, et al. 2012. The rice $\mathrm{HGW}$ gene encodes a ubiquitin-associated (UBA) domain protein that regulates heading date and grain weight. PLoS One 7: e34231.

Li Y, Fan C, Xing Y, Jiang Y, Luo L, Sun L, et al. 2011. Natural variation in GS5 plays an important role in regulating grain size and yield in rice. Nat. Genet. 43: 1266-1269.

Lu L, Shao D, Qiu X, Sun L, Yan W, Zhou X, et al. 2013. Natural variation and artificial selection in four genes determine grain shape in rice. New Phytol. 200: 1269-1280.

Mao H, Sun S, Yao J, Wang C, Yu S, Xu C, et al. 2010. Linking differential domain functions of the GS3 protein to natural variation of grain size in rice. Proc. Natl. Acad. Sci. U.S.A. 107: 19579-19584.

Nagata K, Ando T, Nonoue Y, Mizubayashi T, Kitazawa N, Shomura A, et al. 2015. Advanced backcross QTL analysis reveals complicated genetic control of rice grain shape in a japonica $\mathrm{x}$ indica cross. Breed. Sci. 65: 
308-318.

Panaud O, Chen X, McCouch SR. 1996. Development of microsatellite markers and characterization of simple sequence length polymorphism (SSLP) in rice (Oryza sativa L.). Mol. Gen. Genet. 252: 597-607.

Shomura A, Izawa T, Ebana K, Ebitani T, Kanegae H, Konishi $\mathrm{S}$, et al. 2008. Deletion in a gene associated with grain size increased yields during rice domestication. Nat. Genet. 40: $1023-1028$.

Song XJ, Huang W, Shi M, Zhu MZ, Lin HX. 2007. A QTL for rice grain width and weight encodes a previously unknown RING-type E3 ubiquitin ligase. Nat. Genet. 39: 623-630.

Song XJ, Kuroha T, Ayano M, Furuta T, Nagai K, Komeda N, et al. 2015. Rare allele of a previously unidentified histone $\mathrm{H} 4$ acetyltransferase enhances grain weight, yield, and plant biomass in rice. Proc. Natl. Acad. Sci. U.S.A. 112: 76-81.

Sun L, Li X, Fu Y, Zhu Z, Tan L, Liu F, et al. 2013. GS6, a member of the GRAS gene family, negatively regulates grain size in rice. J. Integr. Plant Biol. 55: 938-949.

Takai T, Adachi S, Taguchi-Shiobara F, Sanoh-Arai Y, Iwasawa N, Yoshinaga S, et al. 2013. A natural variant of NAL1, selected in high-yield rice breeding programs, pleiotropically increases photosynthesis rate. Sci. Rep. 3:
2149.

Wang S, Wu K, Yuan Q, Liu X, Liu Z, Lin X, et al. 2012. Control of grain size, shape and quality by OsSPL16 in rice. Nat. Genet. 44: 950-954.

Xie X, Jin F, Song MH, Suh JP, Hwang HG, Kim YG, et al. 2008. Fine mapping of a yield-enhancing QTL cluster associated with transgressive variation in an Oryza sativa x O. rufipogon cross. Theor. Appl. Genet. 116: 613-622.

Yan S, Zou G, Li S, Wang H, Liu H, Zhai G, et al. 2011. Seed size is determined by the combinations of the genes controlling different seed characteristics in rice. Theor. Appl. Genet. 123: 1173-1181.

Yano M, Sasaki T. 1997. Genetic and molecular dissection of quantitative traits in rice. Plant Mol. Biol. 35: 145-153.

Ying JZ, Gao JP, Shan JX, Zhu MZ, Shi M, Lin HX. 2012. Dissecting the genetic basis of extremely large grain shape in rice cultivar 'JZ1560'. J. Genet. Genom. 39: 325-333.

Yoon DB, Kang KH, Kim HJ, Ju HG, Kwon SJ, Suh JP, et al. 2006. Mapping quantitative trait loci for yield components and morphological traits in an advanced backcross population between Oryza grandiglumis and the $O$. sativa japonica cultivar Hwaseongbyeo. Theor. Appl. Genet. 112: 1052-1062. 